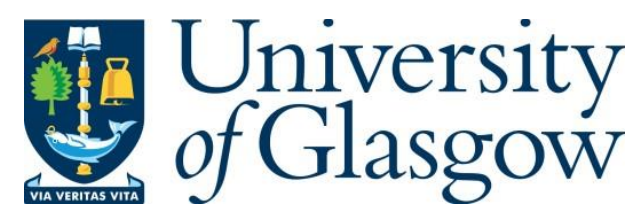

Heidari, H. (2018) Magnetoelectronics: Electronic skins with a global attraction. Nature Electronics, 1(11), pp. 578-579.

There may be differences between this version and the published version. You are advised to consult the publisher's version if you wish to cite from it.

http://eprints.gla.ac.uk/173400/

Deposited on: 4 December 2018

Enlighten - Research publications by members of the University of Glasgow http://eprints.gla.ac.uk 


\section{Magnetoelectronics: Electronic skins with a global attraction}

Magnetic-field sensors integrated on electronic skins can provide an artificial magnetoception that relies only on geomagnetic fields.

\section{Hadi Heidari}

Magnetoelectronic devices exploit both the charge and the spin of an electron in order to provide functionality. They are of use in emerging applications such as human-machine interfaces, memory storage, bioinspired neuromorphic computing, healthcare imaging and diagnostic technologies. Human-machine interfaces, for example, can be realised by exploiting the small magnetic fields that are produced by the electrical activity of the human brain and skeletal muscles ${ }^{1}$ (Fig. 1a). Alternatively, brain-like computing that exhibits tuneable non-linear dynamics and controlled stochasticity can be developed by leveraging novel physical magnetoelectronic effects ${ }^{2}$ (Fig. 1b). And portable medical diagnostics (Fig. 1c) and biomedical imaging ${ }^{3,4}$ can been created through the integration of miniaturised magnetic-based sensors in biochip platforms.

With biomedical applications, bulky magnetoelectronics devices are typically used. However, for approaches that require a compliant and stable interface between the user and the device, such as wearable or implantable health monitors, this can be problematic. Devices based on flexible, stretchable and compliant materials - often referred to as imperceptible devices - offer an attractive alternative, and are expected to lead to electronic-skins that provide a seamless integration scheme. The development of such imperceptible magnetoelectronics also provides the intriguing possibility of expanding the capabilities of humans. In particular, electronic-skin magnetoelectronic devices can enable artificial magnetoception - the ability to sense and respond to magnetic fields ${ }^{5}$. Writing in Nature Electronics, Gilbert Cañón Bermúdez, Denys Makarov and colleagues have now shown that flexible magnetoelectronics devices can be used to create artificial magnetoception that is based solely on the interaction between an imperceptible device and geomagnetic fields ${ }^{6}$ (Fig. 1d).

The researchers - who are based at Helmholtz-Zentrum Dresden-Rossendorf - created a platform to embed a magnetoelectronic compass into a highly compliant functional electronic-skin. The electronic-skin was prepared on 6- $\mu \mathrm{m}$-thin polymeric foils and accommodates magnetic field sensors based on the anisotropic magnetoresistance effect. The response of these compliant sensors was tailored to be linear and to possess a maximum sensitivity around the Earth's magnetic field by arranging the sensors in a Wheatstone bridge configuration. With this setup, Makarov and colleagues were able to demonstrate artificial magnetoception for humans that relies only on the Earth's magnetic field, which is around 40-60 $\mu \mathrm{T}$. Only few animals can derive positional information just from the Earth's magnetic field. For example, organisms such as zebrafish and medaka ${ }^{7}$ possess an internal compass that allows them to orient themselves and maintain a consistent direction of travel.

Flexible magnetoelectronics for artificial magnetoception has been explored before ${ }^{5}$. However, the previous implementations required the use of permanent magnets, as they have to operate at magnetic fields in the $\mathrm{mT}$ range. Makarov and colleagues avoid the use of permanent magnets, thus simplifying the devices for use on human skin. Furthermore, previous efforts used magnetic sensors based on the giant magnetoresistance (GMR) effect, whereas in this latest approach the sensors are based on the anisotropic magnetoresistance (AMR) effect. Although the AMR principle supplies a lower output signal level than competitive GMR approach, the level is more than enough for e-skin compass where it brings down the cost due to the simple fabrication process. This development in AMR technology have made AMR even more competitive with the GMR with reasonably good temperature stability. 
To test the capabilities of their compasses, Makarov and colleagues used them in navigation tasks. The electronic-skins were attached to a person's index finger and, in an open-air experiment, it was shown that they could indicate the person's orientation. The researchers also demonstrated that the electronic-skin compasses could be used to create interactive devices for virtual and augmented reality applications. In particular, the magnetosensitive skins were used in the touchless control of virtual units in a game engine. Furthermore, they showed that their devices can operate with no loss of functionality even under a bending radius of $150 \mu \mathrm{m}$.

In order to develop versatile magnetoelectronics, it will important to focus on devices that do not require permanent magnets, as the work of Makarov and colleagues illustrates. Eliminating the need for such magnets can provide a route to virtual or augmented reality systems that are neither spatially confined nor externally stimulated. And the ability to translate a real frame of reference and orientation, such as the geomagnetic field, into the virtual world, could help bridge the gap between virtual and real experiences.

\section{Hadi Heidari}

Microelectronics Lab (meLAB), School of Engineering, University of Glasgow, UK

e-mail: hadi.heidari@glasgow.ac.uk 


\section{References}

1 Boto, E. et al. Nature 555, 657, (2018).

2 Torrejon, J. et al. Nature 547, 428, (2017).

3 Xiong, Q. et al. Nature Communications 9, 1743, (2018).

4 Lei, K. et al. "IEEE J Solid-St Circuits" 52, 284-297, (2017).

5 Melzer, M. et al. Nature Communications 6, 6080, (2015).

6 Cañón Bermúdez, G. S., Fuchs , H., Bischoff, L., Fassbender, J. \& Makarov, D. Nature Electronics X, XX-XX, (2018).

7 Myklatun, A. et al. Nature Communications 9, 802, (2018).
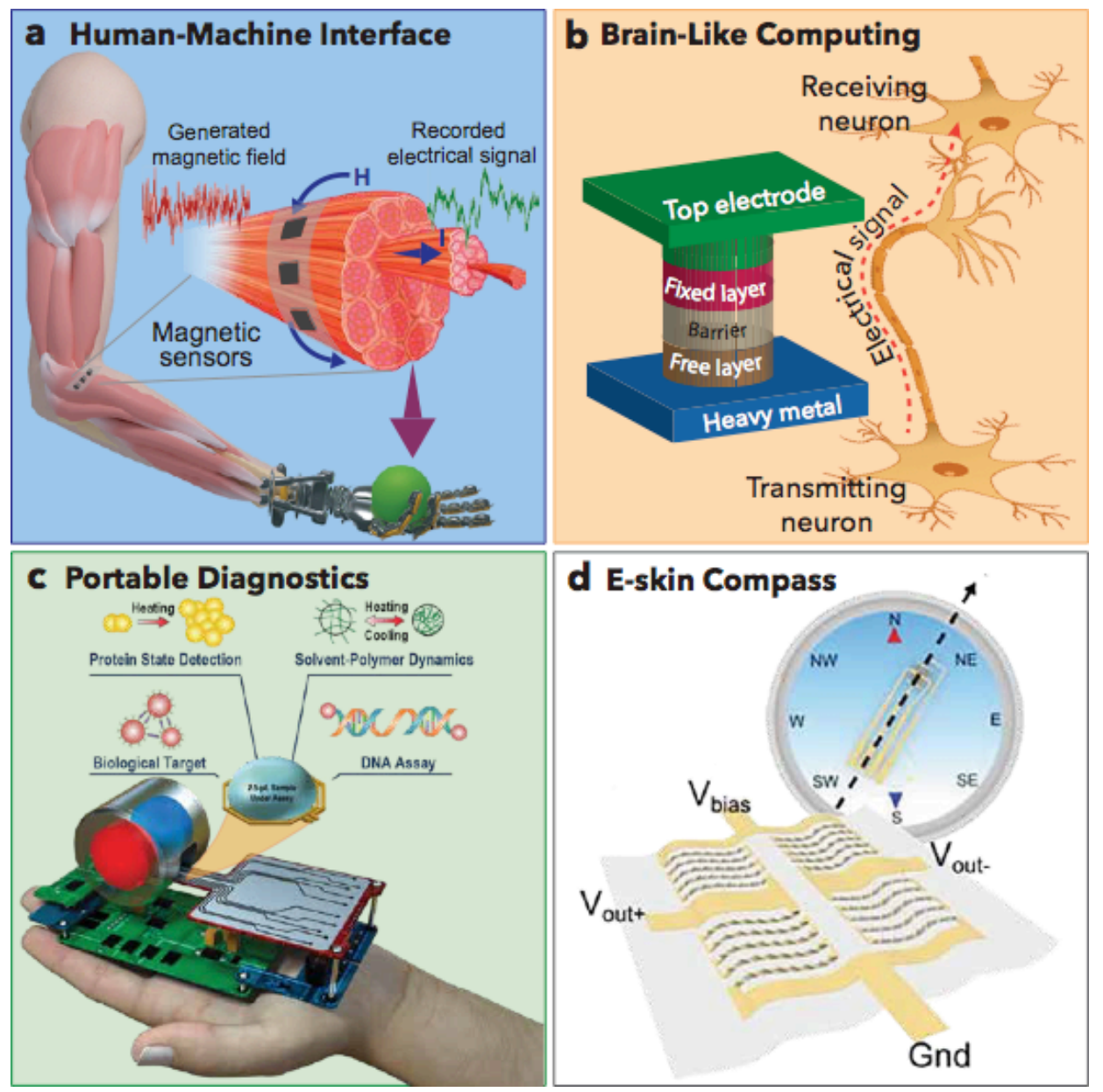

Fig. 1 Emerging magnetoelectronic devices. a, Human-machine interface by exploiting wearable and implantable magnetic sensors. b, Empowering futuristic neuromorphic brain-like computing using nanoscale oscillators. Panel $\mathbf{c}$ adapted from ref. ${ }^{7}$, c, Flexible magnetoelectronics for electronic-skin compasses ${ }^{7}$. Nature Publishing Group. d, Miniaturising magnetic-based biosensors for portable medical diagnostics. 